\title{
RAPID BLEEDING REGION DETECTION IN WIRELESS CAPSULE ENDOSCOPY VIDEOS
}

\begin{abstract}
Professor, Christhujoythi Institute of Technology, sreebommy@gmail.com

Wireless Capsule Endoscopy (WCC) is a medical imaging technique used to examine parts of the gastrointestinal tract. Computer aided detection is used to increase the speed of detection, better performance and reduce the time. Before finding the bleeding regions the edge regions are first detected and removed. Both the edge and the bleeding regions will share the same Hue value and the luminance should be same for the bleeding and the non -bleeding regions. We use a canny edge detector operator for detecting the edge regions in $L$ channel. Canny edge detector is used to detect more edge pixels and preserve more bleeding pixels based up on canny edge algorithm. This method in edge removal algorithm includes edge detection, edge dilation and edge masking. After the removal of edges, those regions are made in to segment through super-pixel segmentation and regions are classified using Artificial Neural Network by Radial Bias Function (RBF).
\end{abstract}

Dr.Sreejamol S.S.

\section{Indexing terms/Keywords}

Canny edge detector, edge detection, super-pixel segmentation

\section{Academic Discipline And Sub-Disciplines}

\author{
Education
}

\section{SUBJECT CLASSIFICATION}

Image processing with wireless endoscopy

\section{TYPE (METHOD/APPROACH)}

\author{
Quasi-Experimental
}

\section{INTRODUCTION}

Wireless Capsule Endoscopy (WCE) has been introduced in 2000 by Given Imaging Ltd. and forms a standard imaging technique for screening the Gastrolntestinal (GI) tract, especially the small intestine. The suspected blood indicator designed by Given Imaging Ltd. can only detect the active bleeding regions in small intestine. Also, this software is known to have insufficient sensitivity and specificity. The performance is measured in terms of three parameters such as sensitivity, specificity and accuracy. High sensitivity means high capability of detecting bleeding frames. High specificity means high capability of avoiding false detection. Accuracy is used to evaluate the overall performance. Since CAD is used to screen for bleeding WCE frames and send them to clinicians for specific examination, sensitivity is more important than specificity and accuracy The Wireless Capsule Endoscopy technique is done using a wireless capsule. The capsule endoscope provides a simple, safe, non-invasive, reliable procedure, well accepted and tolerated by the patient, which avoids any sedation, surgery or radiation exposure. In order to increase the speed and performance and to reduce the time computer aided detection technologies are employed. Here the WCE images are captured using SB pillcam. In this paper a new method for rapid bleeding detection is proposed. Section 2 describes the methodology and section 3 shows the proposed WCE detection algorithm and the implemented results are shown in section 4.

\section{METHODOLOGY}

A new method that can detect bleeding regions from WCE video more effectively and efficiently. For this method the edge pixels are taken in account. Since edge pixels and bleeding pixels share similar hue, where hue represents the actual wavelength of color, ie RED color. In edge regions red intensities of color are more just like the bleeding regions, due to the presence of blood. Traditional algorithms often mistake edge pixels for bleeding pixels. In a typical WCE image, the most distinguishable feature between edge pixels and other non-bleeding pixels is the luminance. The bleeding pixels and non-bleeding pixels (except edge pixels) normally have similar luminance and differ in redness. Therefore the bleeding pixels can't be removed, so the edge regions are first detected and removed. So the images are first converted from RGB space to CIE lab space. Canny edge detector is used because it can detect more edge regions and can preserve more bleeding regions. Through edge masking the intensities of the weak edges pixels are assigned as zero and are removed.

\section{PROPOSED WCE EDGE DETECTION ALGORITHM}

The proposed WCE system includes the removal of edge. The images are preprocessed and it includes Frame Conversion, and De-noising of image. Figure 1 shows the proposed WCE Edge detection. In pre-processing, video is converted as frames. The size of a film frame varies, depending on the still film format or the motion picture film format. A film frame or video frame is one of the many still images which compose the complete moving picture. The video will be read and convert the video as a frames. The converted frame will be saved and the process of elimination of uninformative regions i.e. (dark or bubbled regions) in a frame can make impact on detection of abnormalities. 
Most probably the WCE images are often noisy. The noises are removed and the process is called denoising. Noise reduction techniques are conceptually very similar, regardless of the image being processed. The median filter is a nonlinear digital filtering technique, often used to remove noise. Before edge detection the WCE images are converted from RGB to CIE Lab space. In this color space we use three components: $L$ is the luminance (brightness or intensity), $a$ and $b$ are respectively red/blue and yellow/blue chrominance. The color similarity between pixels can be measured in the CIE Lab space as uniform changes of components in CIE Lab correspond to uniform changes in perceived color.

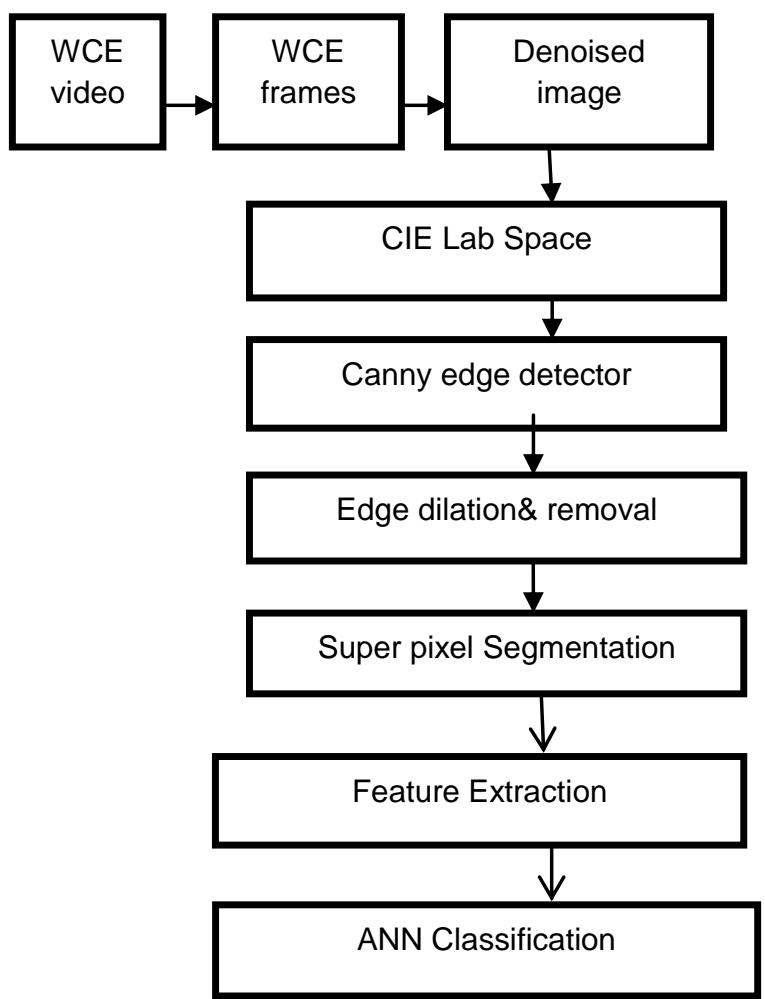

Fig 1: IBlock diagram of proposed WCE Edge detection method

The Canny Edge Detection Algorithm can be explained as follows. The edge pixels are detected using canny edge algorithm. The canny edge algorithm can be broken down into five steps

- Apply Gaussian filter to smooth the image in order to remove the noise

$$
H_{i j}=\frac{1}{2 \pi \sigma^{2}} \exp \left(-\frac{(i-k-1)^{2}+(j-k-1)^{2}}{2 \sigma^{2}}\right)
$$

Find the intensity gradients of the image

$$
\mathrm{G}=\sqrt{ }\left(G x^{2}+G y^{2}\right)
$$

$\Theta=\tan ^{2}(G y, G x)$

\section{Where Gx, Gy is the intensity gradient along horizontal and vertical direction.}

- To get rid of spurious response to edge detection apply non-maximum suppression.

- Use double threshold values to determine potential edges.

- $\quad$ Track edge by hysteresis and finalize the detection of edges by suppressing all the other edges that are weak and not connected to strong edges.

After edge detection, those regions are dilated using mathematical morphological method. For edge dilation two sets of input is needed. $A$ is the image to be dilated. $B$ is a set of coordinate points known as a structuring element (also known as a kernel) which determines the precise effect of the dilation on the input image.

$$
A \oplus B=\{z \mid B z \cap A=\varnothing\}
$$

This equation is based on obtaining the reflection of $B$ about its origin and shifting this reflection by $z$. The dilation of $A$ by $B$ then is the set of all displacements, $z$, such that B and A overlap by at least one pixel. Thus enlarged images of edges are obtained. The following are the steps to remove the edges. 
Convert the WCE image from RGB space to CIE Lab space for the L Channel.

2. Detect the edge regions using canny edge detector.

3. Dilate the detected regions using mathematical morphological method

4. Locate the edge regions by masking, where the intensities of pixels are set to zeros.

\section{EXPERIMENTAL RESULTS}

The edge pixels or the regions from a bleeding WCE video are detected and removed using different process. The results of those processes in WCE images are shown below. This section includes video file uploading shown in 2(a), its frame output shown in 2 (b). These frames are decomposed into RGB components shown in 2 (c). The Denoised output is shown in 2(d). Before edge detection the images are converted from RGB to CIE Lab space shown in 2 (e). The canny edge output is shown in $2(\mathrm{f})$. Figure 2 (g) shows its dilated output and edge removed output is shown in figure 2 (h). Finally figure 2 (i) shows the output of Super pixel segmentation.
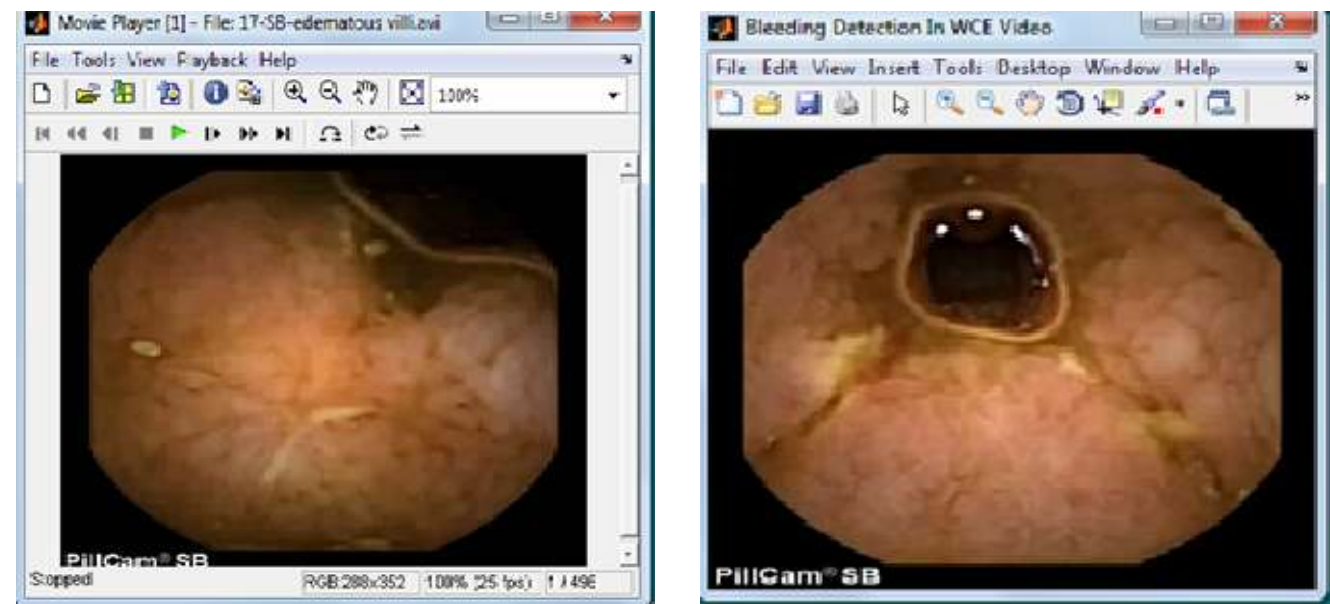

Fig (2):2 (a)uploaded vedio file 2(b) frame conversion
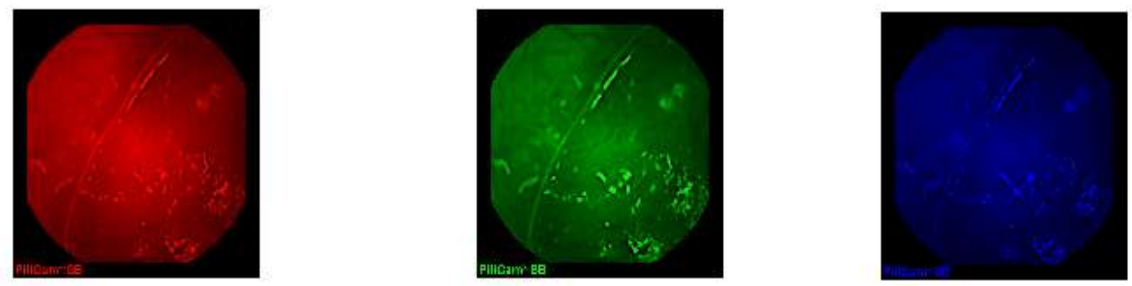

Fig (2): (c)RGB Color components

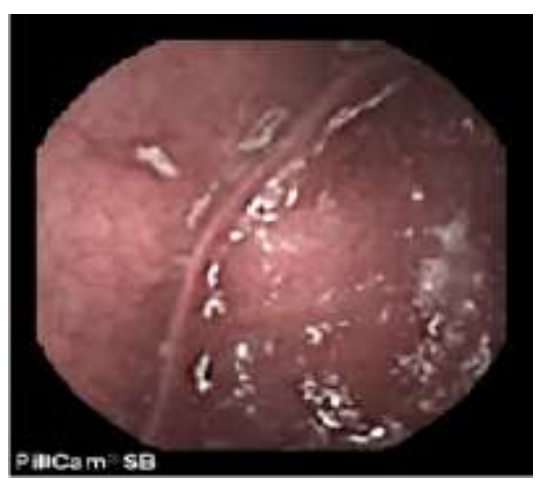

2(d)Denoised Image

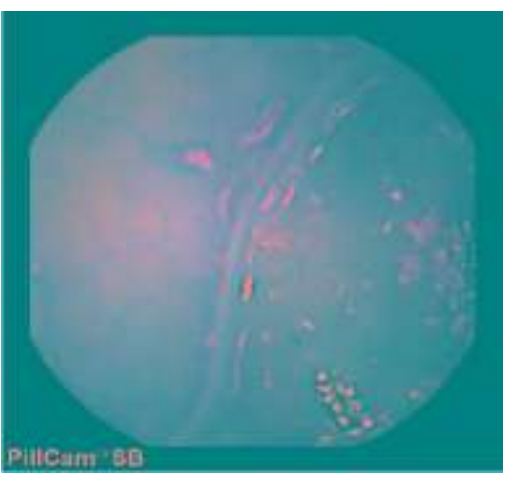

2(e) ) CIE Lab image

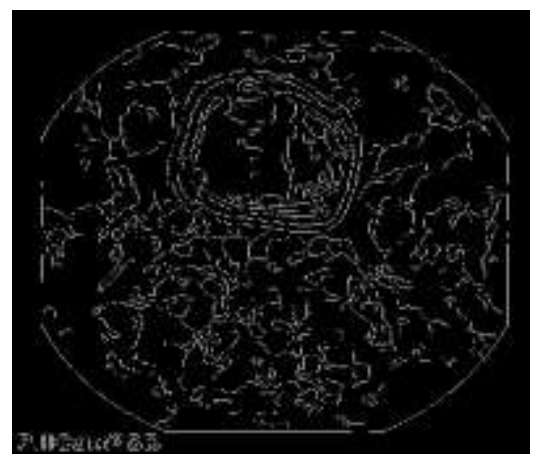

2(f)Canny edge detected image 


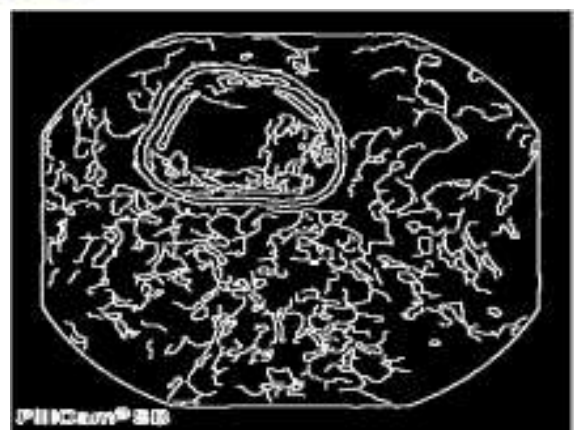

2 (g) Dilated image

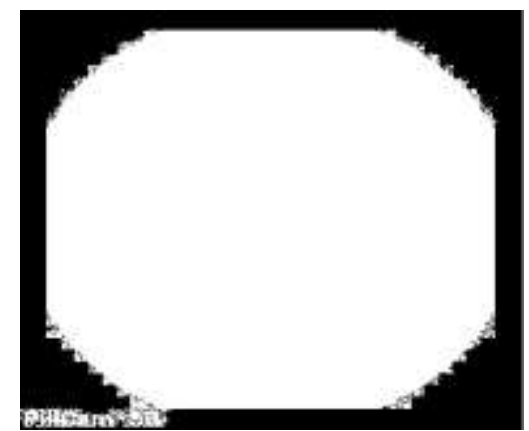

2(h)Edge removed Images

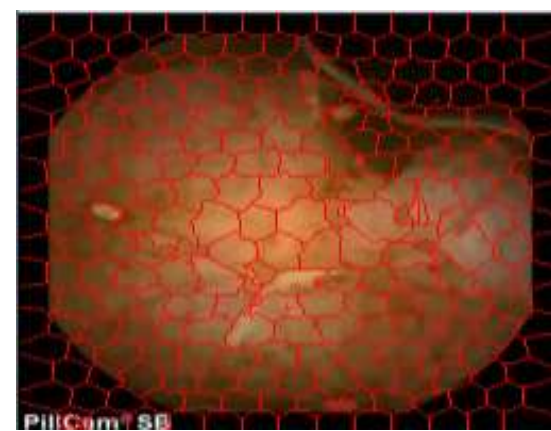

2(i)Super pixel segmented images

Fig (2): experimental results from (d)-(i)

\section{CONCLUSION AND FUTUREWORK}

The edge regions are detected and removed using canny edge detector, which can detect more edge regions so that more bleeding regions can be preserved than any other edge detection methods. Thus well defined edge regions are detected and are removed through masking. In the future those edge removed regions are segmented through super-pixel segmentation and classified as bleeding and non bleeding regions using artificial neural network by using RBF algorithm and compares with other ANN algorithms and finally the performance are measured in terms of accuracy, specificity and sensitivity.

\section{ACKNOWLEDGMENTS}

Our thanks to the experts who have contributed towards development of the template.

\section{REFERENCES}

1. Ali, A., Santisi, J. M., \& Vargo, J. 2004, Video capsule endoscopy: A voyage beyond the end of the scope. Cleveland Clinic journal of medicine, .

2. Iddan, G., Meron, G., Glukhovsky, A., \& Swain, P. 2000, Wireless capsule endoscopy. Nature.

3. G. Zuckerman, C. Prakash, M. Askin, and B. Lewis,2000, AGA technical review on the evaluation and management of occult and obscure gas-trointestinal bleeding," Gastroenterology. Modeling and Simulation Design. AK Peters Ltd.

4. Canny, J.1986, A computational approach to edge detection. IEEE Transactions on pattern analysis and machine intelligence.

5. N. M., \& Eisen, G. M. ,2010), 10 years of capsule endoscopy: An update. Expert review of gastroenterology \& hepatologY.

6. R. C. Gonzalez and R. E. Woods, 2006,Digital Image Processing,, 3rd ed. Up- per Saddle River, NJ, USA: Prentice-Hall.

7. Rajeev, V. R., \& SS, S. M. (2013). FCM Algorithm for Medical Image Segmentation Using HMRF. IJESRT..

8. Liangpunsakul, S., Mays, L., \& Rex, D. K. 2003, Performance of Given suspected blood indicator. The Americanjournaolfgastroenterology.

9. Fu, Y., Mandal, M., \& Guo, G. 2011, Bleeding region detection in WCE images based on color features and neural network. In Circuits and Systems .

\section{Author' biography with Photo}

Dr. SREEJA MOLE S.S, Professor in Christhu Jyothi Institute of Technology and Science and previously worked as a Principal of Pankajakasthuri College of Engineering and Technology was conferred Doctorate Degree in the specialization of ICT from Anna University, Chennai. She graduated in BE - Electronics and Communication Engineering and completed her Post Graduation in Communication Systems with first class distinction. She has over 14.6 years of experience in the field of academia. She started her illustrious career at the Noorul Islam College of Engineering as a Lecturer and was then promoted as Assistant Professor in the Department of Electronics and Communications Engineering. Her commitment in the field of research is reflected in her contribution of more than 26 International Publications. Her area of research includes the digital image processing, computer networks and neural networks. She had convened numerous Workshops/Seminars/National and International conferences and is actively involved in the Spoken tutorial IIT coordinator, QIP, Women empowerment, AISHE and is the member of ISTE, IETE, IE and ACM. She had authored the book "Transmission Lines and Wave Guides" with Dr. L. Ganesan published by Tata Mc- Graw Hill and is in the progress of publishing a book in the field of "Electromagnetic Field and Advanced Digital System Design" with Dr. L. Ganesan. 